\title{
Some Properties of Prequasi Normed Generalized de La Vallée Poussin's Mean Sequence Space
}

\author{
Awad A. Bakery $\mathbb{D}^{1,2}$ and Mustafa M. Mohammed ${ }^{10}{ }^{1,3}$ \\ ${ }^{1}$ University of Jeddah, College of Science and Arts at Khulis, Department of Mathematics, Jeddah, Saudi Arabia \\ ${ }^{2}$ Department of Mathematics, Faculty of Science, Ain Shams University, P.O. Box 1156, Cairo 11566, Abbassia, Egypt \\ ${ }^{3}$ Department of Statistics, Faculty of Science, Sudan University of Science \& Technology, Khartoum, Sudan
}

Correspondence should be addressed to Mustafa M. Mohammed; mustasta@gmail.com

Received 9 December 2019; Revised 24 February 2020; Accepted 16 April 2020; Published 11 May 2020

Academic Editor: Raúl E. Curto

Copyright ( $) 2020$ Awad A. Bakery and Mustafa M. Mohammed. This is an open access article distributed under the Creative Commons Attribution License, which permits unrestricted use, distribution, and reproduction in any medium, provided the original work is properly cited.

In this article, we study some topological properties of the multiplication operator on generalized de La Vallée Poussin's mean sequence space equipped with the prequasi norm and the prequasi operator ideal generated by $s$-numbers and this sequence space.

\section{Introduction}

All through the article, by $L(U, V)$, we signify the space of all bounded linear transformations between arbitrary Banach spaces $U$ and $V$; if $U=V$, we mark $L(U)$. We will denote $F(V), \Psi(V)$, and $L_{c}(V)$ for the space of all finite rank, approximable, and compact transformations on $V$, respectively. The set is composed of nonnegative integers $(\mathbb{N})$ and real numbers $(\mathbb{R})$. Moreover, $\mathbb{C}^{\mathbb{N}}, \ell_{\infty}, C_{0},\left(s_{r}(A)\right),\left(d_{r}(A)\right)$, and $\left(\alpha_{r}(A)\right)$ will denote the space of complex, bounded, null, r-th $s$-numbers [1], r-th Kolmogorov, and approximation sequences, respectively, where $A \in L(U, V)$. The multiplication operators and operator ideals have a wide field of mathematics in functional analysis, for instance, in eigenvalue distributions theorem, fixed point theorem, geometry of Banach spaces, and spectral theory. Singh and Kumar [2] investigated the connection between the composition operators and the multiplication operators on $L_{q}$-spaces. They proved the equivalence between the composition operator $A \in L_{c}\left(L_{q}(U ; \mathbb{C})\right)$ and $B_{\beta} \in L_{c}\left(L_{q}(U ; \mathbb{C})\right)$, where $B_{\beta}$ is the multiplication operator induced by $\beta=d v B^{-1} / d v$. The roots of the multiplication operators are contained in the spectral theory. According to Hilbert space theorem, there is a unitary equivalence between a multiplication operator and each normal operator on a separable Hilbert space. For more subtleties on multiplication operators, see [2-7]. In view of sequence spaces, Mursaleen and Noman [8] investigated some difference sequence spaces under compact matrix transformation. The multiplication operators belong to $L\left(\ell_{\varphi}\right)$, where $\varphi$ is an Orlicz function, studied by Komal and Gupta [9]. Furthermore, Komal et al. [10] explained the multiplication operators in $L\left(\left(\operatorname{ces}_{q}\right)_{\|.\|}\right)$, for $q \in(1, \infty)$ with the Luxemburg norm $\|$.$\| . In the operator ideal theory, the scalar sequence$ space is sometimes used to define operator ideals in the class of Banach spaces or Hilbert spaces, since the ideal $L_{c}(U)$ is generated by $C_{0}$ and $\left(d_{r}(A)\right)$. A Banach space $U$ is called simple [11], if there is one and only one nontrivial closed ideal in $L(U)$. The quasi-ideals $S_{\ell_{q}}^{\text {app }}$, where $q \in(0, \infty)$, is investigated by Pietsch [11]. Also, he proved that $\ell_{2}$ and $\ell_{1}$ generated the ideals of Hilbert Schmidt operators between Hilbert spaces and of nuclear operators, respectively. $\mathrm{He}$ also investigated the sufficient condition on $\ell_{q}$ so that $F(\bar{U}, V)=S_{\ell_{q}}^{\text {app }}(U, V)$ and $L\left(\ell_{q}\right)$ is simple Banach space. Pietsch [12] studied the smallness of $S_{\ell_{q}}^{\text {app }}$. For any infinite dimensional Banach spaces $U$ and $V$, Makarov and Faried [13] gave the sufficient conditions for which $S_{\ell_{r}}^{\text {app }}$ is strictly contained in $S_{\ell_{m}}^{\text {app }}$, for every $m$ $>r>0$. Bakery [14] introduced some properties of $S_{V(\gamma, p)}^{\text {app }}$, where $V(\gamma, p)$ is the generalized de La Vallée Poussin's mean 
sequence space. Some properties of $s$-type Orlicz-Lorentz sequence spaces are examined by Mohiuddine and Raj [15]. Faried and Bakery [16] introduced a generalization of the usual classes of operator ideal which is prequasi operator ideal. They explained some results of $S_{\operatorname{ces}(q)}$ and $S_{\ell}$. We introduce in this paper the concept of prequasi norm on $V(\gamma, p)$, give the conditions on $V(\gamma, p)$ equipped with the prequasi norm to construct Banach space, and study the necessity and sufficient conditions on $V(\gamma, p)$ so that the multiplication operator defined on $V(\gamma, p)$ is bounded, invertible, approximable, closed range, and Fredholm operator. We investigate the sufficient conditions on the class $S_{V(\gamma, p)}$ to form small, simple, closed Banach prequasi operator ideal. Also, we examine the strict inclusion relation between $S_{V(\gamma, p)}$ and $S_{E}^{\lambda}$ (the class of all bounded linear operators whose sequence of eigenvalues belongs to $V(\gamma, p))$.

\section{Definitions and Preliminaries}

We will denote $e_{j}=(0,0, \cdots, 1,0,0, \cdots)$, with 1 in the $j^{\text {th }}$ position for every $j \in \mathbb{N}$.

Lemma 1 (see [11]). For $T \in L(V, W)$, if $T \notin \Psi(V, W)$, then there are operators $B \in L(W)$ and $G \in L(V)$ with $B T G e_{i}=e_{i}$ for each $i \in \mathbb{N}$.

Theorem 2 (see [11]). If $W$ is a Banach space with $\operatorname{dim}(W)$ $=\infty$, then

$$
F(W) \varsubsetneqq \Psi(W) \varsubsetneqq L_{c}(W) \varsubsetneqq L(W) .
$$

Definition 3 (see [17]). An operator $D \in L(V)$ is called Fredholm if it satisfies $\operatorname{dim}(\operatorname{ker} D)<\infty$, $\operatorname{dim}(R(D))^{c}<\infty$, and $D$ has closed range, where $(R(D))^{c}$ denotes the complement of the range $D$.

Let $\left(\gamma_{k}\right) \in\left(\mathbb{R}^{+}\right)^{\mathbb{N}}$, where $\gamma_{0}=1, \gamma_{k+1} \geq \gamma_{k}, \gamma_{k+1} \leq \gamma_{k}+1$, for all $k \in \mathbb{N}, \lim _{k \rightarrow \infty} \gamma_{k}=\infty$ and $\left(p_{i}\right) \in\left(\mathbb{R}^{+}\right)^{\mathbb{N}}$ with $p_{i} \geq 1$, for all $i \in \mathbb{N}$. Simsek et al. [18] defined the generalized de La Vallée Poussin's mean sequence space as follows:

$$
\begin{aligned}
V(\gamma, p)= & \left\{x=\left(x_{i}\right) \in \mathbb{C}^{\mathbb{N}}: \exists \eta>0 \text { with } \sigma(\eta x)<\infty\right\} \\
& \text { where } \sigma(x)=\sum_{i=0}^{\infty}\left(\frac{\sum_{k \in G_{i}}\left|x_{k}\right|}{\gamma_{i}}\right)^{p_{i}},
\end{aligned}
$$

and $G_{i}=\left[i-\gamma_{i}+1, i\right]$, for $i \in \mathbb{N}$. The space $(V(\gamma, p),\|\cdot\|)$, where

$$
\|x\|=\inf \left\{\eta>0: \sigma\left(\frac{x}{\eta}\right) \leq 1\right\}
$$

is a Banach space. When $\left(p_{n}\right) \in \ell_{\infty}$, it is clear that

$$
V(\gamma, p)=\left\{\left(x_{i}\right) \in \mathbb{C}^{\mathbb{N}}: \sum_{i=0}^{\infty}\left(\frac{\sum_{k \in G_{i}}\left|x_{k}\right|}{\gamma_{i}}\right)^{p_{i}}<\infty\right\} .
$$

For more details on $V(\gamma, p)$, see $[19,20]$.

\section{Remark 4.}

(1) If $\gamma_{i}=i+1$, for every $i \in \mathbb{N}$, then $V(\gamma, p)=\operatorname{ces}\left(\left(p_{n}\right)\right)$ examined by Sanhan and Suantai [21]

(2) If $\gamma_{i}=i+1$ and $p_{i}=p$, for all $i \in \mathbb{N}$, then $V(\gamma, p)=$ $\operatorname{ces}_{p}$. Some authors [22-24] investigated various sorts of Cesáro summable sequence spaces

Definition 5 (see [25]). A class of linear sequence spaces $X$ is called a special space of sequences (sss) if

(1) $e_{j} \in X$ for each $j \in \mathbb{N}$

(2) for $u=\left(u_{j}\right) \in \mathbb{C}^{\mathbb{N}}, v=\left(v_{j}\right) \in X$ and $\left|u_{j}\right| \leq\left|v_{j}\right|$ for all $j$ $\in \mathbb{N}$, then $u \in X$

(3) for $\left(u_{j}\right)_{j=0}^{\infty} \in X$, then $\left(u_{[j / 2]}\right)_{j=0}^{\infty} \in X$, wherever $[j / 2]$ means the integral part of $j / 2$

Theorem 6 (see [14]). $V(\gamma, p)$ is a (sss), if

(a1) $\left(p_{k}\right)$ is increasing and $\left(p_{k}\right) \in \ell_{\infty}$ with $p_{0}>1$

(a2) $\left(\gamma_{k}\right) \in\left(\mathbb{R}^{+}\right)^{\mathbb{N}}$, where $\gamma_{0}=1, \gamma_{k+1} \geq \gamma_{k}, \gamma_{k+1} \leq \gamma_{k}+1$, for all $k \in \mathbb{N}, \lim _{k \rightarrow \infty} \gamma_{k}=\infty$ and $\left(\gamma_{k}^{-1}\right) \in \ell_{\left(p_{k}\right)}$ are satisfied

Definition 7 (see [25]). A subclass of (sss) is called a premodular (sss) if there is a function $\sigma: X \longrightarrow[0, \infty)$ verifying the conditions

(i) $\sigma(v) \geq 0$ for all $v \in X$ and $\sigma(v)=0 \Leftrightarrow v=\theta$, here $\theta$ is the zero element of $X$

(ii) there is $L \geq 1$ with $\sigma(\eta v) \leq L|\eta| \sigma(v)$ for each $v \in X$, and $\eta \in \mathbb{C}$

(iii) there is $K \geq 1, \sigma(u+v) \leq K(\sigma(u)+\sigma(v))$ for all $u$, $v \in X$

(iv) for $\left|u_{i}\right| \leq\left|v_{i}\right|$ for all $i \in \mathbb{N}$, then $\sigma\left(\left(u_{i}\right)\right) \leq \sigma\left(\left(v_{i}\right)\right)$

(v) for some $K_{0} \geq 1, \sigma\left(\left(u_{i}\right)\right) \leq \sigma\left(\left(u_{[i / 2]}\right)\right) \leq K_{0} \sigma\left(\left(u_{i}\right)\right)$

(vi) for all $u=\left(u_{j}\right)_{j=o}^{\infty} \in X$ and $\varepsilon>0$, there is $s \in \mathbb{N}$ with $\sigma\left(\left(u_{j}\right)_{j=s}^{\infty}\right)<\varepsilon$

(vii) there is $\xi>0$ such that $\sigma(\eta, 0,0,0, \cdots) \geq \xi|\eta| \sigma(1,0$, $0,0, \cdots)$ for each $\eta \in \mathbb{C}$

Theorem 8 (see [14]). If conditions (a1) and (a2) are verified, then $(V(\gamma, p))_{\sigma}$ is a premodular (sss), with $\sigma(x)=\sum_{j=0}^{\infty}$ $\left(\sum_{k \in G_{j}}\left|x_{k}\right| / \gamma_{j}\right)^{p_{j}}$ for each $x \in V(\gamma, p)$. The usual classes of operator ideal generalized to the prequasi operator ideal.

Definition 9 (see [25]). A function $f: \Phi \longrightarrow[0, \infty)$ is called a prequasi norm on the ideal $\Phi$ if it satisfies the following: 
(1) If $A \in \Phi(U, V)$, then $f(A) \geq 0$ and $f(A)=0$ if and only if $A=0$

(2) There is $M \geq 1$ with $f(\eta A) \leq M|\eta| f(A)$, for each $A \in$ $\Phi(U, V)$ and $\eta \in \mathbb{C}$

(3) There is $K \geq 1$ such that $f\left(A_{1}+A_{2}\right) \leq K\left[f\left(A_{1}\right)+f\right.$ $\left.\left(A_{2}\right)\right]$, for every $A_{1}, A_{2} \in \Phi(U, V)$

(4) There is $C \geq 1$, if $A \in L\left(U_{0}, U\right), P \in \Phi(U, V)$ and $R \in L\left(V, V_{0}\right)$ then $f(R P A) \leq C\|R\| f(P)\|A\|$, where $U_{0}$ and $V_{0}$ are normed spaces

Notations 10 (see [16]).

$$
\begin{aligned}
S_{E}:= & \left\{S_{E}(U, V) ; U \text { and } V \text { are Banach Spaces }\right\}, \\
& \text { with } S_{E}(U, V):=\left\{A \in L(U, V):\left(s_{i}(A)\right)_{i=0}^{\infty} \in E\right\} . \\
S_{E}^{\mathrm{app}}:= & \left\{S_{E}^{\mathrm{app}}(U, V) ; U \text { and } V \text { are Banach Spaces }\right\}, \\
& \text { with } S_{E}^{\mathrm{app}}(U, V):=\left\{A \in L(U, V):\left(\alpha_{i}(A)\right)_{i=0}^{\infty} \in E\right\} . \\
S_{E}^{K o l}:= & \left\{S_{E}^{K o l}(U, V) ; U \text { and } V \text { are Banach Spaces }\right\}, \\
& \text { with } S_{E}^{K o l}(U, V):=\left\{T \in L(U, V):\left(d_{i}(A)\right)_{i=0}^{\infty} \in E\right\} .
\end{aligned}
$$

Theorem 11 (see [16]). Let $E_{\mathrm{Q}}$ be a premodular (sss), then the function $g(T)=\mathrm{Q}\left(s_{i}(T)\right)_{i=0}^{\infty}$ be a prequasi norm on $S_{E_{\mathrm{Q}}}$.

During this paper, $\left(p_{i}\right) \in\left(\mathbb{R}^{+}\right)^{\mathbb{N}}$ with $\left(p_{i}\right) \in \ell_{\infty}$ and the inequality [26] $\left|a_{j}+b_{j}\right|^{p_{j}} \leq H\left(\left|a_{j}\right|^{p_{j}}+\left|b_{j}\right|^{p_{j}}\right)$, where $H=2^{h-1}$, $h=\sup _{j} p_{j}$, and $p_{j} \geq 1$ for all $j \in \mathbb{N}$, will be used.

\section{Main Results}

We introduce a generalization of the usual norm, the concept of prequasi norm on $V(\gamma, p)$. We investigate the sufficient conditions on $V(\gamma, p)$ equipped with a prequasi norm to form Banach space.

Definition 12. Assume $X$ is (sss). If there is a function $\sigma: X$ $\longrightarrow[0, \infty)$ satisfying the conditions

(i) $\sigma(v) \geq 0$ for all $v \in X$ and $\sigma(v)=0 \Leftrightarrow v=\theta$

(ii) there is $L \geq 1$ with $\sigma(\eta v) \leq L|\eta| \sigma(v)$ for every $v \in X$, and $\eta \in \mathbb{C}$

(iii) there is $K \geq 1$, we have $\sigma(u+v) \leq K(\sigma(u)+\sigma(v))$ for each $u, v \in X$

The space $X_{\sigma}$ is called prequasi normed (sss). If $X$ is complete with $\sigma$, hence $X_{\sigma}$ is called a prequasi Banach (sss). We express the following two theorems without verification, since they are clear.

Theorem 13. $X$ is a prequasi norm (sss), if it is quasi norm (sss).
Theorem 14. Every premodular (sss) is prequasi normed (sss).

Theorem 15. If conditions (a1) and (a2) are verified, then $(V(\gamma, p))_{\mathrm{Q}}$ is a prequasi Banach (sss), with $\mathrm{Q}(x)=\sum_{i=0}^{\infty}$ $\left(\sum_{j \in G_{i}}\left|x_{j}\right| / \gamma_{i}\right)^{p_{i}}$ for all $x \in V(\gamma, p)$.

Proof. Let the conditions be satisfied, then from Theorem 8 , the space $(V(\gamma, p))_{0}$ is premodular (sss). From Theorem 14, we have $(V(\gamma, p))_{\mathrm{e}}$ which is a prequasi normed (sss). To show that $(V(\gamma, p))_{\mathrm{Q}}$ is a prequasi Banach (sss), assume $x^{n}=\left(x_{k}^{n}\right)_{k=0}^{\infty}$ be a Cauchy sequence in $(V(\gamma, p))_{\mathrm{Q}}$. Therefore, for all $\varepsilon \in(0,1)$, there is $n_{0} \in \mathbb{N}$ such that for all $n, m \geq n_{0}$, one has

$$
\mathrm{\varrho}\left(x^{n}-x^{m}\right)=\sum_{i=0}^{\infty}\left(\frac{\sum_{j \in G_{i}}\left|x_{j}^{n}-x_{j}^{m}\right|}{\gamma_{i}}\right)^{p_{i}}<\varepsilon^{h} .
$$

Hence for $n, m \geq n_{0}$ and $j \in \mathbb{N}$, we get

$$
\left|x_{j}^{n}-x_{j}^{m}\right|<\varepsilon
$$

So $\left(x_{j}^{m}\right)$ is a Cauchy sequence in $\mathbb{C}$ for fixed $j \in \mathbb{N}$; this gives $\lim _{m \rightarrow \infty} x_{j}^{m}=x_{j}^{0}$ for fixed $j \in \mathbb{N}$. Hence, $\mathrm{\varrho}\left(x^{n}-x^{0}\right)<\varepsilon^{h}$, for all $n \geq n_{0}$. Finally, to prove that $x^{0} \in V(\gamma, p)$, we have

$$
\mathrm{\varrho}\left(x^{0}\right)=\mathrm{\varrho}\left(x^{0}-x^{n}+x^{n}\right) \leq H\left(\mathrm{\varrho}\left(x^{n}-x^{0}\right)+\mathrm{\varrho}\left(x^{n}\right)\right)<\infty,
$$

so $x^{0} \in V(\gamma, p)$. This completes the proof.

Corollary 16. Let $1<p<\infty$, then $\left(\text { ces }_{p}\right)_{\mathrm{Q}}$ be a prequasi Banach (sss), with $\mathrm{\varrho}(x)=\sum_{i=0}^{\infty}\left(\sum_{j=0}^{i}\left|x_{j}\right| / i+1\right)^{p}$ for all $x \in$ ces $_{p}$.

\section{Multiplication Operator on Prequasi Normed (sss)}

We define here a multiplication operator on $V(\gamma, p)$ with a prequasi norm and investigate the sufficient and necessary conditions on the multiplication operator to become bounded, closed range operator, approximable, invertible, and Fredholm.

Definition 17. If $\eta \in \mathbb{C}^{\mathbb{N}}, \eta \in \ell_{\infty}$ and $X_{\mathrm{o}}$ is a prequasi normed (sss). An operator $T_{\eta}$ is called multiplication operator if $T_{\eta}$ $: X \longrightarrow X$, where $T_{\eta} y=\eta y=\left(\eta_{k} y_{k}\right)_{k=0}^{\infty}$, for all $y \in X$. When $T_{\eta} \in L(X), T_{\eta}$ is called the multiplication operator induced by $\eta$.

Theorem 18. Let $\alpha \in \mathbb{C}^{\mathbb{N}}$, the conditions (a1) and (a2) be satisfied, hence $\alpha \in \ell_{\infty}$ if and only if $T_{\alpha} \in L\left((V(\gamma, p))_{\mathrm{Q}}\right)$, with $\mathrm{Q}(x)=\sum_{i=0}^{\infty}\left(\sum_{j \in G_{i}}\left|x_{j}\right| / \gamma_{i}\right)^{p_{i}}$ for every $x \in(V(\gamma, p))_{\mathrm{Q}}$.

Proof. Let the conditions be satisfied and $\alpha \in \ell_{\infty}$. Therefore, there is $D>0$ with $\left|\alpha_{k}\right| \leq D$, for each $k \in \mathbb{N}$. For $x \in$ 
$(V(\gamma, p))_{\mathrm{e}}$, we have

$$
\begin{aligned}
\mathrm{Q}\left(T_{\alpha} x\right) & =\mathrm{\varrho}(\alpha x)=\mathrm{\varrho}\left(\left(\alpha_{k} x_{k}\right)_{k=0}^{\infty}\right)=\mathrm{Q}\left(\left(\left|\alpha_{k} \| x_{k}\right|\right)_{k=0}^{\infty}\right) \\
& =\sum_{i=0}^{\infty}\left(\frac{\sum_{j \in G_{i}}\left|\alpha_{j}\right|\left|x_{j}\right|}{\gamma_{i}}\right)^{p_{i}} \leq \sum_{i=0}^{\infty}\left(\frac{\sum_{j \in G_{i}} C\left|x_{j}\right|}{\gamma_{i}}\right)^{p_{i}} \\
& \leq L \mathrm{Q}(x),
\end{aligned}
$$

where $L=\sup _{i} D^{p_{i}}$; this implies that $T_{\alpha} \in L\left((V(\gamma, p))_{\mathrm{Q}}\right)$. Conversely, let $T_{\alpha} \in L\left((V(\gamma, p))_{\mathrm{e}}\right)$ to show that $\alpha \in \ell_{\infty}$. For, if $\alpha \notin \ell_{\infty}$, hence for all $n \in \mathbb{N}$, there are $i_{n} \in \mathbb{N}$ with $\alpha_{i_{n}}>n$. We have

$$
\begin{aligned}
\mathrm{Q}\left(T_{\alpha} e_{i_{n}}\right) & =\mathrm{Q}\left(\alpha e_{i_{n}}\right)=\mathrm{Q}\left(\left(\alpha_{j}\left(e_{i_{n}}\right)_{j}\right)_{j=0}^{\infty}\right) \\
& =\sum_{i=0}^{\infty}\left(\frac{\sum_{j \in G_{i}}\left|\alpha_{j}\right|\left(e_{i_{n}}\right)_{j}}{\gamma_{i}}\right)^{p_{i}}=\sum_{i=0}^{\infty}\left(\frac{\left|\alpha_{i_{n}}\right|}{\gamma_{i}}\right)^{p_{i}} \\
& >\sum_{i=0}^{\infty}\left(\frac{n}{\gamma_{i}}\right)^{p_{i}} \geq \inf _{i} n^{p_{i}} \mathrm{Q}\left(e_{i_{n}}\right)=n^{p_{0}} \mathrm{Q}\left(e_{i_{n}}\right) .
\end{aligned}
$$

This shows that $T_{\alpha} \notin L\left((V(\gamma, p))_{\mathrm{e}}\right)$. So, $\alpha \in \ell_{\infty}$.

Theorem 19. If $\alpha \in \mathbb{C}^{\mathbb{N}}$ and $(V(\gamma, p))_{\mathrm{e}}$ is a prequasi normed (sss), with $\mathrm{Q}(x)=\sum_{i=0}^{\infty}\left(\sum_{j \in G_{i}}\left|x_{j}\right| / \gamma_{i}\right)^{p_{i}}$ for each $x \in(V(\gamma, p))_{\mathrm{Q}}$. Hence, $\left|\alpha_{j}\right|=1$ for each $j \in \mathbb{N}$ if and only if $T_{\alpha}$ is an isometry.

Proof. Suppose $\left|\alpha_{j}\right|=1$, for every $j \in \mathbb{N}$. Therefore,

$$
\begin{aligned}
\mathrm{\varrho}\left(T_{\alpha} x\right) & =\mathrm{\varrho}(\alpha x)=\mathrm{\varrho}\left(\left(\alpha_{j} x_{j}\right)_{j=0}^{\infty}\right)=\sum_{i=0}^{\infty}\left(\frac{\sum_{j \in G_{i}}\left|\alpha_{j}\right|\left|x_{j}\right|}{\gamma_{i}}\right)^{p_{i}} \\
& =\sum_{i=0}^{\infty}\left(\frac{\sum_{j \in G_{i}}\left|x_{j}\right|}{\gamma_{i}}\right)^{p_{i}}=\mathrm{\varrho}(x),
\end{aligned}
$$

for all $x \in(V(\gamma, p))_{\mathrm{e}}$. Hence, $T_{\alpha}$ is an isometry. Conversely, let $\left|\alpha_{n}\right|<1$ for some $n=n_{0}$. Then,

$$
\begin{aligned}
\mathrm{Q}\left(T_{\alpha} e_{n_{0}}\right) & =\mathrm{\varrho}\left(\alpha e_{n_{0}}\right)=\mathrm{\varrho}\left(\left(\alpha_{j}\left(e_{n_{0}}\right)_{j}\right)_{j=0}^{\infty}\right) \\
& =\sum_{i=0}^{\infty}\left(\frac{\sum_{j \in G_{i}}\left|\alpha_{j}\right|\left|\left(e_{n_{0}}\right)_{j}\right|}{\gamma_{i}}\right)^{p_{i}} \\
& =\sum_{i=0}^{\infty}\left(\frac{\alpha_{n_{0}}}{\gamma_{i}}\right)^{p_{i}}<\sum_{i=0}^{\infty}\left(\frac{1}{\gamma_{i}}\right)^{p_{i}}<\mathrm{\varrho}\left(e_{n_{0}}\right) .
\end{aligned}
$$

Also, if $\left|\alpha_{n_{0}}\right|>1$, then we get $\mathrm{Q}\left(T_{\alpha} e_{n_{0}}\right)>\mathrm{Q}\left(e_{n_{0}}\right)$. Therefore, we have a contradiction in the two cases. So, $\left|\alpha_{j}\right|=1$, for each $j \in \mathbb{N}$.
Theorem 20. If $\alpha \in \mathbb{C}^{\mathbb{N}}$, the conditions (a1) and (a2) are satisfied and $T_{\alpha} \in L\left((V(\gamma, p))_{\mathrm{e}}\right)$, where $\rho(x)=\sum_{r=0}^{\infty}\left(\sum_{j \in G_{r}}\left|x_{j}\right| /\right.$ $\left.\gamma_{r}\right)^{p_{r}}$ for all $x \in(V(\gamma, p))_{0}$. Then, $T_{\alpha} \in \Psi\left((V(\gamma, p))_{0}\right)$ if and only if $\left(\alpha_{n}\right)_{n=0}^{\infty} \in C_{0}$.

Proof. Let $T_{\alpha}$ be an approximable operator. Hence, $T_{\alpha} \in$ $L_{c}\left((V(\gamma, p))_{\mathrm{Q}}\right)$. To prove that $\left(\alpha_{r}\right)_{r=0}^{\infty} \in C_{0}$, assume $\left(\alpha_{r}\right)_{r=0}^{\infty} \notin$ $C_{0}$; hence, there is $\delta>0$ with $B_{\delta}=\left\{r \in \mathbb{N}:\left|\alpha_{r}\right| \geq \delta\right\}$ which is an infinite set. Suppose $d_{1}, \cdots d_{2}, \cdots, d_{n}, \cdots$ be in $B_{\delta}$. Then, $\left\{e_{d_{n}}: d_{n} \in B_{\delta}\right\}$ is an infinite bounded set in $(V(\gamma, p))_{\mathrm{e}}$. We have

$$
\begin{aligned}
& \mathrm{\varrho}\left(T_{\alpha} e_{d_{n}}-T_{\alpha} e_{d_{m}}\right) \\
& \quad=\mathrm{Q}\left(\alpha e_{d_{n}}-\alpha e_{d_{m}}\right) \\
& =\mathrm{\varrho}\left(\left(\alpha_{k}\left(\left(e_{d_{n}}\right)_{k}-\left(e_{p_{m}}\right){ }_{k}\right)\right)_{k=0}^{\infty}\right) \\
& \quad=\sum_{i=0}^{\infty}\left(\frac{\sum_{j \in G_{i}}\left|\alpha_{j}\left(\left(e_{d_{n}}\right)_{j}-\left(e_{p_{m}}\right)_{j}\right)\right|}{\gamma_{i}}\right)^{p_{i}} \\
& \quad \geq \sum_{i=0}^{\infty}\left(\frac{\left.\sum_{j \in G_{i}}\left|\delta\left(\left(e_{d_{n}}\right)_{j}-\left(e_{p_{m}}\right)_{j}\right)\right|\right)^{p_{i}}}{\gamma_{i}}\right) \\
& \quad \geq\left(\inf _{n} \delta^{p_{n}}\right) \sum_{i=0}^{\infty}\left(\frac{\sum_{j \in G_{i}}\left|\left(\left(e_{d_{n}}\right)_{j}-\left(e_{p_{m}}\right)_{j}\right)\right|}{\gamma_{i}}\right)^{p_{i}} \\
& \quad=\left(\inf _{n} \delta^{p_{n}}\right) \rho\left(e_{d_{n}}-e_{d_{m}}\right),
\end{aligned}
$$

for all $d_{n}, d_{m} \in B_{\delta}$. This proves $\left\{e_{d_{n}}: d_{n} \in B_{\delta}\right\} \in \ell_{\infty}$ which cannot have a convergent subsequence under $T_{\alpha}$. Therefore, this gives $T_{\alpha} \notin L_{c}\left((V(\gamma, p))_{\mathrm{Q}}\right)$, so $T_{\alpha} \notin \Psi\left((V(\gamma, p))_{\mathrm{Q}}\right)$; this implies a contradiction. Therefore, $\lim _{n \rightarrow \infty} \alpha_{n}=0$. Conversely, suppose $\lim _{n \rightarrow \infty} \alpha_{n}=0$. Hence, for all $\delta>0$, the set $B_{\delta}=$ $\left\{n \in \mathbb{N}:\left|\alpha_{n}\right| \geq \delta\right\}$ is finite. We have

$$
\left((V(\gamma, p))_{\mathrm{e}}\right)_{B_{\delta}}=\left\{x=\left(x_{n}\right) \in(V(\gamma, p))_{\mathrm{e}}: n \in B_{\delta}\right\},
$$

which is a finite dimensional space for all $\delta>0$. Hence, $T_{\alpha} \mid\left((V(\gamma, p))_{\mathrm{Q}}\right)_{B_{\delta}}$ is a finite rank operator. Define $\alpha_{i} \in \mathbb{C}^{\mathbb{N}}$ as follows

$$
\left(\alpha_{i}\right)_{j}= \begin{cases}\alpha_{j}, & j \in B_{1 / i}, \\ 0, & \text { otherwise. }\end{cases}
$$


It is clear that since $T_{\alpha_{i}}$ is a finite rank operator, then the space $\left((V(\gamma, p))_{\mathrm{Q}}\right)_{B_{1 / i}}$ is finite dimensional for each $i \in \mathbb{N}$. Therefore, by using conditions (a1) and (a2), we have

$$
\begin{aligned}
\mathrm{Q}( & \left.\left(T_{\alpha}-T_{\alpha_{n}}\right) x\right) \\
= & \mathrm{Q}\left(\left(\left(\alpha_{j}-\left(\alpha_{n}\right)_{j}\right) x_{j}\right)_{j=0}^{\infty}\right) \\
= & \sum_{i=0, i \in B_{1 / n}}^{\infty}\left(\frac{\sum_{j \in G_{i}}\left|\left(\alpha_{j}-\left(\alpha_{n}\right)_{j}\right) x_{j}\right|}{\gamma_{i}}\right)^{p_{i}} \\
& +\sum_{i=0, i \notin B_{1 / n}}^{\infty}\left(\frac{\sum_{j \in G_{i}}\left|\left(\alpha_{j}-\left(\alpha_{n}\right)_{j}\right) x_{j}\right|}{\gamma_{i}}\right)^{p_{i}} \\
= & \sum_{i=0, i \in B_{1 / n}}^{\infty}\left(\frac{\sum_{j \in G_{i} j \notin B_{1 / n}}\left|\alpha_{j} x_{j}\right|}{\gamma_{i}}\right)^{p_{i}} \\
& +\sum_{i=0, i \notin B_{1 / n}}^{\infty}\left(\frac{\sum_{j \in G_{i} j \notin B_{1 / n}}\left|\alpha_{j} x_{j}\right|}{\gamma_{i}}\right)^{p_{i}} \\
< & \frac{1}{n} \sum_{i=0}^{\infty}\left(\frac{\sum_{j \in G_{i}}\left|x_{j}\right|}{\gamma_{i}}\right)^{p_{i}}=\frac{1}{n} \mathrm{Q}(x) .
\end{aligned}
$$

This proves that $\left\|T_{\alpha}-T_{\alpha_{n}}\right\| \leq 1 / n$. So, $T_{\alpha}$ is a limit of finite rank operators. This finishes the proof.

Theorem 21. If $\alpha \in \mathbb{C}^{\mathbb{N}}$, the conditions (a1) and (a2) are satisfied and $T_{\alpha} \in L\left((V(\gamma, p))_{\mathrm{e}}\right)$, where $\rho(x)=\sum_{i=0}^{\infty}\left(\sum_{j \in G_{i}}\left|x_{j}\right| /\right.$ $\left.\gamma_{i}\right)^{p_{i}}$ for all $x \in(V(\gamma, p))_{\mathrm{Q}}$. Then, $T_{\alpha} \in L_{c}\left((V(\gamma, p))_{\mathrm{Q}}\right)$ if and only if $\left(\alpha_{n}\right)_{n=0}^{\infty} \in C_{0}$.

Proof. It is clear so omitted.

Corollary 22. If conditions (a1) and (a2) are satisfied, we have

$$
L_{c}\left((V(\gamma, p))_{\mathrm{e}}\right) \varsubsetneqq L\left((V(\gamma, p))_{\mathrm{e}}\right),
$$

with $\mathrm{\varrho}(x)=\sum_{i=0}^{\infty}\left(\sum_{j \in G_{i}}\left|x_{j}\right| / \gamma_{i}\right)^{p_{i}}$ for each $x \in V(\gamma, p)$.

Proof. Since the multiplication operator induced by $\alpha=$ $(1,1$,$) is I \in L\left((V(\gamma, p))_{\mathrm{e}}\right)$, therefore $I \notin L_{c}\left((V(\gamma, p))_{\mathrm{e}}\right)$.

Theorem 23. Let $(V(\gamma, p))_{\mathrm{e}}$ be a prequasi Banach (sss), with $\left.T_{\alpha} \in L((V(\gamma, p)))_{\mathrm{e}}\right)$ and $\mathrm{\varrho}(x)=\sum_{i=0}^{\infty}\left(\sum_{j \in G_{i}}\left|x_{j}\right| \gamma_{i}\right)^{p_{i}}$ for each $x \in V(\gamma, p)$. Hence, $\alpha$ is bounded away from zero on $\operatorname{ker}(\alpha)^{c}$ if and only if $T_{\alpha}$ has closed range.

Proof. Let the sufficient conditions be satisfied. Therefore, there is $\varepsilon>0$ such that $\left|\alpha_{j}\right| \geq \varepsilon$, for every $j \in \operatorname{ker}(\alpha)^{c}$. To show that $R\left(T_{\alpha}\right)$ is closed, suppose $q$ be a limit point of $R\left(T_{\alpha}\right)$. Hence, there is $T_{\alpha} x_{j}$ in $(V(\gamma, p))_{\mathrm{e}}$, for all $j \in \mathbb{N}$ such that $\lim _{j \rightarrow \infty} T_{\alpha} x_{j}=q$. Clearly, the sequence $T_{\alpha} x_{j}$ is a Cauchy sequence. Therefore, since $\rho$ is nondecreasing, one can see

$$
\begin{aligned}
& \mathrm{Q}\left(T_{\alpha} x_{n}-T_{\alpha} x_{m}\right) \\
&=\mathrm{Q}\left(\left(\alpha_{j}\left(x_{n}\right)_{j}-\alpha_{j}\left(x_{m}\right)_{j}\right)_{j=0}^{\infty}\right) \\
&=\sum_{i=0}^{\infty}\left(\frac{\sum_{j \in G_{i}}\left|\alpha_{j}\left(x_{n}\right)_{j}-\alpha_{j}\left(x_{m}\right)_{j}\right|}{\gamma_{i}}\right)^{p_{i}} \\
&=\sum_{i=0, i \in \operatorname{ker}(\alpha)^{c}}^{\infty}\left(\frac{\sum_{j \in G_{i}}\left|\alpha_{j}\left(x_{n}\right)_{j}-\alpha_{j}\left(x_{m}\right)_{j}\right|}{\gamma_{i}}\right)^{p_{i}} \\
& \quad+\sum_{i=0, i \notin \mathrm{ker}(\alpha)^{c}}^{\infty}\left(\frac{\sum_{j \in G_{i}}\left|\alpha_{j}\left(x_{n}\right)_{j}-\alpha_{j}\left(x_{m}\right)_{j}\right|}{\gamma_{i}}\right)^{p_{i}} \\
& \sum_{i=0, i \in \operatorname{ker}(\alpha)^{c}}^{\infty}\left(\frac{\sum_{j \in G_{i}}\left|\alpha_{j}\left(x_{n}\right)_{j}-\alpha_{j}\left(x_{m}\right)_{j}\right|}{\gamma_{i}}\right)^{p_{i}} \\
&\left.=\sum_{i=0}^{\infty}\left(\frac{\sum_{j \in G_{i}}\left|\alpha_{j}\left(y_{n}\right)_{j}-\alpha_{j}\left(y_{m}\right)_{j}\right|}{\gamma_{i}}\right)^{p_{i}}\right)^{p_{i}}=\mathrm{Q}\left(\varepsilon\left(y_{n}-y_{m}\right)\right), \\
&> \sum_{i=0}^{\infty}\left(\frac{\sum_{j \in G_{i}}\left|\varepsilon\left(y_{n}\right)_{j}-\varepsilon\left(y_{m}\right)_{j}\right|}{\gamma_{i}}\right.
\end{aligned}
$$

where

$$
\left(y_{n}\right)_{j}= \begin{cases}\left(x_{n}\right)_{j}, & j \in \operatorname{ker}(\alpha)^{c}, \\ 0, & j \notin \operatorname{ker}(\alpha)^{c} .\end{cases}
$$

Hence, $\left(y_{n}\right)$ is a Cauchy sequence in $(V(\gamma, p))_{\mathrm{e}}$ since $(V(\gamma, p))_{\mathrm{Q}}$ is complete. So, there is $x \in(V(\gamma, p))_{\mathrm{e}}$ with $\lim _{n \rightarrow \infty} y_{n}=x$. Since $T_{\alpha}$ is continuous, hence $\lim _{n \rightarrow \infty} T_{\alpha} y_{n}=$ $T_{\alpha} x$. But $\lim _{n \rightarrow \infty} T_{\alpha} x_{n}=\lim _{n \rightarrow \infty} T_{\alpha} y_{n}=q$. Therefore, $T_{\alpha} x=$ $q$. Hence, $q \in R\left(T_{\alpha}\right)$. This gives $R\left(T_{\alpha}\right)$ is closed. Let the necessary condition be satisfied; this means $R\left(T_{\alpha}\right)$ be closed. Therefore, $T_{\alpha}$ is bounded away from zero on $\left((V(\gamma, p))_{\rho}\right)_{\operatorname{ker}(\alpha)^{c}}$, i.e., there is $\varepsilon>0$ such that $\mathrm{Q}\left(T_{\alpha} x\right) \geq \mathrm{Q}(\varepsilon x)$, for all $x \in$ $\left((V(\gamma, p))_{\mathrm{e}}\right)_{\mathrm{ker}(\alpha)^{c}}$. Let $D=\left\{j \in \operatorname{ker}(\alpha)^{c}:\left|\alpha_{j}\right|<\varepsilon\right\}$. If $D \neq \phi$, then for $n_{0} \in D$, since $\varrho$ is nondecreasing, we have

$$
\begin{aligned}
\mathrm{Q}\left(T_{\alpha} e_{n_{0}}\right) & \left.=\mathrm{Q}\left(\left(\alpha_{j}\left(e_{n_{0}}\right)_{j}\right)\right)_{j=0}^{\infty}\right)=\mathrm{Q}\left(\left(\left|\alpha_{j}\right|\left|\left(e_{n_{0}}\right)_{j}\right|\right)_{j=0}^{\infty}\right) \\
& =\sum_{i=0}^{\infty}\left(\frac{\sum_{j \in G_{i}}\left|\alpha_{j}\right|\left|\left(e_{n_{0}}\right)_{j}\right|}{\gamma_{i}}\right)^{p_{i}}<\sum_{i=0}^{\infty}\left(\frac{\sum_{j \in G_{i}} \varepsilon\left|\left(e_{n_{0}}\right)_{j}\right|}{i+1}\right)^{p_{i}} \\
& =\mathrm{Q}\left(\varepsilon e_{n_{0}}\right) .
\end{aligned}
$$


This gives a contradiction. Therefore, $D=\phi$ with $\left|\alpha_{j}\right|$ $\geq \varepsilon$, for each $j \in \operatorname{ker}(\alpha)^{c}$. This completes the proof.

Theorem 24. If $\alpha \in \mathbb{C}^{\mathbb{N}}$ and $(V(\gamma, p))_{\mathrm{e}}$ is a prequasi Banach (sss), with $\mathrm{Q}(x)=\sum_{i=0}^{\infty}\left(\sum_{j \in G_{i}}\left|x_{j}\right| / \gamma_{i}\right)^{p_{i}}$ for each $x \in V(\gamma, p)$. Hence, there is $b>0$ and $B>0$ such that $b<\alpha_{j}<B$; for each $j \in \mathbb{N}$ if and only if $T_{\alpha} \in L\left((V(\gamma, p))_{0}\right)$ is invertible.

Proof. Assume that the sufficient condition be verified. Define $\eta \in \mathbb{C}^{\mathbb{N}}$ by $\eta_{j}=1 / \alpha_{j}$. Then by Theorem $18, T_{\alpha}$ and $T_{\eta}$ are bounded linear operators. Also, $T_{\alpha} \cdot T_{\eta}=T_{\eta} \cdot T_{\alpha}=I$. Hence, $T_{\eta}$ is the inverse of $T_{\alpha}$. Conversely, suppose the necessary condition be satisfied. Therefore, $R\left(T_{\alpha}\right)=\left((V(\gamma, p))_{\rho}\right)_{\mathbb{N}}$. Hence, $R\left(T_{\alpha}\right)$ is closed. From Theorem 23, there is $b>0$ so that $\left|\alpha_{j}\right| \geq b$, for all $j \in \operatorname{ker}(\alpha)^{c}$. Now, $\operatorname{ker}(\alpha)=\phi$; otherwise, $\alpha_{n_{0}}=0$, for some $n_{0} \in \mathbb{N}$, we have $e_{n_{0}} \in \operatorname{ker}\left(T_{\alpha}\right)$. Since ker $\left(T_{\alpha}\right)$ is trivial, this gives a contradiction. Therefore, $\left|\alpha_{j}\right| \geq$ $b$, for each $j \in \mathbb{N}$. From Theorem 18, since $T_{\alpha}$ is bounded, there is $B>0$ so that $\left|\alpha_{j}\right| \leq B$, for every $j \in \mathbb{N}$. Therefore, we have showed that $b \leq\left|\alpha_{j}\right| \leq B$, for all $j \in \mathbb{N}$.

Theorem 25. If $(V(\gamma, p))_{\mathrm{Q}}$ is a prequasi Banach (sss), with $T_{\alpha} \in L\left((V(\gamma, p))_{\mathrm{e}}\right)$ and $\rho(y)=\sum_{i=0}^{\infty}\left(\sum_{j \in G_{i}}\left|y_{j}\right| \gamma_{i}\right)^{p_{i}}$ for every $y \in V(\gamma, p)$. Hence, $T_{\alpha}$ be Fredholm operator if and only if (i) $\operatorname{ker}(\alpha)$ be a finite subset of $\mathbb{N}$. (ii) $\left|\alpha_{j}\right| \geq \varepsilon$, for each $j \in \operatorname{ker}(\alpha)^{c}$.

Proof. Let $T_{\alpha}$ be Fredholm. Assume $\operatorname{ker}(\alpha)$ be an infinite subset of $\mathbb{N}$, then $e_{j} \in \operatorname{ker}\left(T_{\alpha}\right)$, for each $j \in \operatorname{ker}(\alpha)$. This gives $\operatorname{dim}\left(\operatorname{ker}\left(T_{\alpha}\right)\right)=\infty$; hence, we have a contradiction. Therefore, $\operatorname{ker}(\alpha)$ be a finite subset of $\mathbb{N}$. From Theorem 23 , condition (ii) follows. On the other hand, let the necessary condition be satisfied. From Theorem 23, condition (ii) shows that $R\left(T_{\alpha}\right)$ is closed and condition (i) gives that $\operatorname{dim}\left(\operatorname{ker}\left(T_{\alpha}\right)\right)<\infty$ and $\operatorname{dim}\left(R\left(T_{\alpha}\right)\right)^{c}<\infty$. This finishes the proof.

\section{Closed Banach Prequasi Ideal}

We study the sufficient conditions on $V(\gamma, p)$ so that the prequasi ideal $S_{V(\gamma, p)}$ are Banach and closed.

Theorem 26. Let $X$ and $Y$ be Banach spaces, (a1) and (a2) be satisfied, then $\left(S_{(V(\gamma, p))}, g\right)$ is a prequasi Banach operator ideal, where $g(T)=\rho\left(\left(s_{n}(T)\right)_{n=0}^{\infty}\right)$ and $\rho(x)=\sum_{i=0}^{\infty}\left(\sum_{j \in G_{i}}\left|x_{j}\right| /\right.$ $\left.\gamma_{i}\right)^{p_{i}}$ for each $x \in V(\gamma, p)$.

Proof. From Theorem 8, the space $(V(\gamma, p))_{\rho}$ is a premodular (sss). Hence, by using Theorem 11, we have the function $g(T)=\rho\left(\left(s_{n}(T)\right)_{n=0}^{\infty}\right)$ that is a prequasi norm on $S_{(V(\gamma, p))_{e}}$. Suppose $\left(T_{j}\right)$ be a Cauchy sequence in $S_{(V(\gamma, p))_{e}}(X, Y)$, then by part (vii) of Definition 7 there is $\xi>0$ and since $S_{(V(\gamma, p))_{\mathrm{e}}}$ $(X, Y) \subseteq L(X, Y)$, we get

$$
\begin{aligned}
g\left(T_{i}-T_{j}\right) & =\sum_{r=0}^{\infty}\left(\frac{1}{\gamma_{r}} \sum_{k \in G_{r}} s_{k}\left(T_{i}-T_{j}\right)\right)^{p_{r}} \\
& \geq \sum_{r=0}^{\infty}\left(\frac{1}{\gamma_{r}}\left\|T_{i}-T_{j}\right\|\right)^{p_{r}} \geq \xi\left\|T_{i}-T_{j}\right\| \sum_{r=0}^{\infty}\left(\frac{1}{\gamma_{r}}\right)^{p_{r}} .
\end{aligned}
$$

Therefore, $\left(T_{j}\right)_{j \in \mathbb{N}}$ be a Cauchy sequence in $L(X, Y)$. Since $L(X, Y)$ is a Banach space, hence there is $T \in L(X, Y)$ with $\lim _{i \rightarrow \infty}\left\|T_{i}-T\right\|=0$. Since $\left(s_{n}\left(T_{i}\right)\right)_{n=0}^{\infty} \in(V(\gamma, p))_{\mathrm{Q}}$ for each $i \in \mathbb{N}$, from parts (ii), (iii), (iv), and (v) of Definition 7 , we have

$$
\begin{aligned}
g(T)= & \sum_{r=0}^{\infty}\left(\frac{1}{\gamma_{r}} \sum_{k \in G_{r}}\left(s_{k}(T)\right)^{p_{r}}=\sum_{r=0}^{\infty}\left(\frac{1}{\gamma_{r}} \sum_{k \in G_{r}}\left(s_{k}\left(T-T_{m}+T_{m}\right)\right)^{p_{r}}\right.\right. \\
\leq & K \sum_{r=0}^{\infty}\left(\frac{1}{\gamma_{r}} \sum_{k \in G_{r}} s_{\left[\frac{k}{2}\right]}\left(T-T_{m}\right)\right)^{p_{r}} \\
& +K \sum_{r=0}^{\infty}\left(\frac{1}{\gamma_{r}} \sum_{k \in G_{r}} s_{\left[\frac{k}{2}\right]}\left(T_{m}\right)\right)^{p_{r}} \\
\leq & K \sum_{r=0}^{\infty}\left(\frac{1}{\gamma_{r}} \sum_{k \in G_{r}}\left\|T_{m}-T\right\|\right)^{p_{r}} \\
& +K K_{0} \sum_{r=0}^{\infty}\left(\frac{1}{\gamma_{r}} \sum_{k \in G_{r}} s_{k}\left(T_{m}\right)\right)^{p_{r}}<\varepsilon .
\end{aligned}
$$

Therefore, $\left(s_{j}(T)\right)_{j=0}^{\infty} \in(V(\gamma, p))_{\rho}$; hence, $T \in S_{(V(\gamma, p))_{e}}$ $(X, Y)$.

Theorem 27. Let $X$ and $Y$ be Banach spaces, (a1) and (a2) be satisfied, then $\left(S_{(V(\gamma, p))_{\mathrm{e}}}, g\right)$ is a prequasi closed operator ideal, where $g(T)=\rho\left(\left(s_{n}(T)\right)_{n=0}^{\infty}\right)$ and $\rho(x)=\sum_{i=0}^{\infty}\left(\sum_{j \in G_{i}}\left|x_{j}\right| \gamma_{i}\right)^{p_{i}}$ for each $x \in V(\gamma, p)$.

Proof. From Theorem 8, the space $(V(\gamma, p))_{\rho}$ is a premodular (sss). Hence, by using Theorem 11, we have the function $g(T)=\rho\left(\left(s_{n}(T)\right)_{n=0}^{\infty}\right)$ that is a prequasi norm on $S_{(V(\gamma, p))_{e}}$. Suppose $T_{j} \in S_{(V(\gamma, p))_{e}}(X, Y)$ for each $j \in \mathbb{N}$ and $\lim _{m \rightarrow \infty} g$ $\left(T_{m}-T\right)=0$. Since $S_{(V(\gamma, p))_{e}}(X, Y) \subseteq L(X, Y)$ and from part (vii) of Definition 7 , we have

$$
\begin{aligned}
g\left(T-T_{m}\right) & =\sum_{r=0}^{\infty}\left(\frac{1}{\gamma_{r}} \sum_{k \in G_{r}} s_{k}\left(T-T_{m}\right)\right)^{p_{r}} \\
& \geq \sum_{r=0}^{\infty}\left(\frac{1}{\gamma_{r}}\left\|T-T_{m}\right\|\right)^{p_{r}} \geq \xi\left\|T-T_{m}\right\| \sum_{r=0}^{\infty}\left(\frac{1}{\gamma_{r}}\right)^{p_{r}} .
\end{aligned}
$$


Hence, $\left(T_{m}\right)_{m \in \mathbb{N}}$ is convergent in $L(X, Y)$. Since $\left(s_{n}\left(T_{i}\right)\right)_{n=0}^{\infty} \in(V(\gamma, p))_{\mathrm{e}}$ for each $i \in \mathbb{N}$, from parts (ii), (iii), (iv), and (v) of Definition 7, we obtain

$$
\begin{aligned}
g(T)= & \sum_{r=0}^{\infty} \frac{1}{\gamma_{r}} \sum_{k \in G_{r}}\left(s_{k}(T)\right)^{p_{r}}=\sum_{r=0}^{\infty} \frac{1}{\gamma_{r}} \sum_{k \in G_{r}}\left(s_{k}\left(T-T_{m}+T_{m}\right)\right)^{p_{r}} \\
\leq & K \sum_{r=0}^{\infty}\left(\frac{1}{\gamma_{r}} \sum_{k \in G_{r}} s_{[k / 2]}\left(T-T_{m}\right)\right)^{p_{r}} \\
& +K \sum_{r=0}^{\infty}\left(\frac{1}{\gamma_{r}} \sum_{k \in G_{r}} s_{[k / 2]}\left(T_{m}\right)\right)^{p_{r}} \\
\leq & K \sum_{r=0}^{\infty}\left(\frac{1}{\gamma_{r}} \sum_{k \in G_{r}}\left\|T_{m}-T\right\|\right)^{p_{r}} \\
& +K K_{0} \sum_{r=0}^{\infty}\left(\frac{1}{\gamma_{r}} \sum_{k \in G_{r}} s_{k}\left(T_{m}\right)\right)^{p_{r}}<\varepsilon .
\end{aligned}
$$
$(X, Y)$.

Therefore, $\left(s_{n}(T)\right)_{n=0}^{\infty} \in(V(\gamma, p))_{\rho}$. Hence, $T \in S_{(V(\gamma, p))_{e}}$

\section{Small and Simple Prequasi Banach Operator Ideal}

We introduce some inclusion relations concerning prequasi operator ideal constructed by the sequence of $s$-numbers and $V(\gamma, p)$.

Theorem 28. If $X, Y$ are infinite dimensional Banach spaces, $\left(p_{r}\right) \in \ell_{\infty},\left(q_{r}\right) \in \ell_{\infty}$ with $1<p_{r}<q_{r}$, and $1 \leq \gamma_{r} \leq v_{r}$ for every $r \in \mathbb{N}$, then

$$
S_{V(\gamma, p)}(X, Y) \varsubsetneqq S_{V(v, q)}(X, Y) \varsubsetneqq L(X, Y) .
$$

Proof. Assume the sufficient conditions be verified and $T \epsilon$ $S_{V(\gamma, p)}(X, Y)$. Therefore, $\left(s_{k}(T)\right) \in V(\gamma, p)$. We have

$$
\sum_{r=0}^{\infty}\left(\frac{1}{v_{r}} \sum_{k \in G_{r}} s_{k}(T)\right)^{q_{r}}<\sum_{r=0}^{\infty}\left(\frac{1}{\gamma_{r}} \sum_{k \in G_{r}} s_{k}(T)\right)^{p_{r}}<\infty .
$$

Hence, $T \in S_{V(v, q)}(X, Y)$. By taking $\left(s_{r}(T)\right)_{r=0}^{\infty}$ such that $\sum_{k \in G_{r}} s_{k}(T)=\gamma_{r}(r+1)^{-1 / p_{r}}$, one can find $T \in L(X, Y)$ so that

$$
\begin{aligned}
\sum_{r=0}^{\infty}\left(\frac{1}{\gamma_{r}} \sum_{k \in G_{r}} s_{k}(T)\right)^{p_{r}} & =\sum_{r=0}^{\infty} \frac{1}{r+1}=\infty, \\
\sum_{r=0}^{\infty}\left(\frac{1}{v_{r}} \sum_{k \in G_{r}} s_{k}(T)\right)^{q_{r}} & \leq \sum_{r=0}^{\infty}\left(\frac{1}{\gamma_{r}} \sum_{k \in G_{r}} s_{k}(T)\right)^{q_{r}} \\
& =\sum_{r=0}^{\infty}\left(\frac{1}{r+1}\right)^{q_{r} / p_{r}}<\infty .
\end{aligned}
$$

Hence, $T \in S_{V(v, q)}(X, Y)$ and $T \notin S_{V(\gamma, p)}(X, Y)$. Obviously, $S_{V(\gamma, p)}(X, Y) \subset L(X, Y)$. By choosing $\left(s_{r}(T)\right)_{r=0}^{\infty}$ such that $\sum_{k \in G_{r}} s_{k}(T)=v_{r}(r+1)^{-1 / q_{r}}$. We have $T \in L(X, Y)$ and $T \notin$ $S_{V(v, q)}(X, Y)$. This finishes the proof.

Corollary 29. If $X, Y$ are infinite dimensional Banach spaces and $1<p<q<\infty$, it is true that

$$
S_{c e s_{p}}(X, Y) \varsubsetneqq S_{c e s_{q}}(X, Y) \varsubsetneqq L(X, Y) .
$$

We investigate the sufficient conditions so that the prequasi Banach operator ideal $S_{V(\gamma, p)}^{a p p}$ is small.

Theorem 30. The prequasi Banach operator ideal $S_{V(\gamma, p)}^{a p p}$ is small, whenever $X, Y$ are infinite dimensional Banach spaces and the conditions (a1) and (a2) are satisfied.

Proof. If the sufficient conditions are verified, therefore the space $\left(S_{V(\gamma, p)}^{\text {app }}, g\right)$ is a prequasi Banach operator ideal, with $g$ $(T)=1 / \eta\left(\sum_{i=0}^{\infty}\left(\gamma_{i}^{-1} \sum_{j \in G_{i}} \alpha_{j}(T)\right)^{p_{i}}\right)^{1 / h}$ and $\eta=\left(\sum_{i=0}^{\infty} \gamma_{i}^{-p_{i}}\right)^{1 / h}$. Suppose that $S_{V(\gamma, p)}^{\text {app }}(X, Y)=L(X, Y)$, so there is $C>0$ so that $g(T) \leq C\|T\|$ for each $T \in L(X, Y)$. From Dvoretzky's theorem [27] for all $m \in \mathbb{N}$, we obtain subspaces $M_{m}$ of $Y$ and quotient spaces $X / N_{m}$ transform onto $\ell_{2}^{m}$ by isomorphisms $A_{m}$ and $H_{m}$ with $\left\|A_{m}\right\|\left\|A_{m}^{-1}\right\| \leq 2$ and $\left\|H_{m}\right\|\left\|H_{m}^{-1}\right\| \leq 2$. If $J_{m}$ is the natural embedding map from $M_{m}$ into $Y$ and $Q_{m}$ is the quotient map from $X$ onto $X / N_{m}$, suppose $v_{n}$ denotes the Bernstein numbers [28], we have

$$
\begin{aligned}
1 & =u_{n}\left(I_{m}\right)=v_{n}\left(A_{m} A_{m}^{-1} I_{m} H_{m} H_{m}^{-1}\right) \\
& \leq\left\|A_{m}\right\| v_{n}\left(A_{m}^{-1} I_{m} H_{m}\right)\left\|H_{m}^{-1}\right\| \\
& =\left\|A_{m}\right\| v_{n}\left(J_{m} A_{m}^{-1} I_{m} H_{m}\right)\left\|H_{m}^{-1}\right\| \\
& \leq\left\|A_{m}\right\| d_{n}\left(J_{m} A_{m}^{-1} I_{m} H_{m}\right)\left\|H_{m}^{-1}\right\| \\
& =\left\|A_{m}\right\| d_{n}\left(J_{m} A_{m}^{-1} I_{m} H_{m} Q_{m}\right)\left\|H_{m}^{-1}\right\| \\
& \leq\left\|A_{m}\right\| \alpha_{n}\left(J_{m} A_{m}^{-1} I_{m} H_{m} Q_{m}\right)\left\|H_{m}^{-1}\right\|,
\end{aligned}
$$

for $0 \leq i \leq m$. Now

$$
\begin{aligned}
\sum_{j \in G_{i}}\left(\gamma_{i}^{-1}\right) & \leq \sum_{j \in G_{i}}\left\|A_{m}\right\| \gamma_{i}^{-1} \alpha_{j}\left(J_{m} A_{m}^{-1} I_{m} H_{m} Q_{m}\right)\left\|H_{m}^{-1}\right\| \Rightarrow 1 \\
& \leq\left\|A_{m}\right\|\left(\gamma_{i}^{-1} \sum_{j \in G_{i}} \alpha_{j}\left(J_{m} A_{m}^{-1} I_{m} H_{m} Q_{m}\right)\right)\left\|H_{m}^{-1}\right\| \Rightarrow 1 \\
& \leq\left(\left\|A_{m}\right\|\left\|H_{m}^{-1}\right\|\right)^{p_{i}}\left(\gamma_{i}^{-1} \sum_{j \in G_{i}} \alpha_{j}\left(J_{m} A_{m}^{-1} I_{m} H_{m} Q_{m}\right)\right)^{p_{i}} .
\end{aligned}
$$


Therefore,

$$
\begin{aligned}
(m+1)^{1 / h} \leq & L\left\|A_{m}\right\|\left\|H_{m}^{-1}\right\| \\
& \cdot\left[\sum_{i=0}^{m}\left(\gamma_{i}^{-1} \sum_{j \in G_{i}} \alpha_{j}\left(J_{m} A_{m}^{-1} I_{m} H_{m} Q_{m}\right)\right)^{p_{i}}\right]^{1 / h} \\
\Rightarrow & \frac{1}{\eta}(m+1)^{1 / h} \leq L\left\|A_{m}\right\|\left\|H_{m}^{-1}\right\| \frac{1}{\eta} \\
& \cdot\left[\sum_{i=0}^{m}\left(\gamma_{i}^{-1} \sum_{j \in G_{i}} \alpha_{j}\left(J_{m} A_{m}^{-1} I_{m} H_{m} Q_{m}\right)\right)^{p_{i}}\right]^{1 / h} \\
\Rightarrow & \frac{1}{\eta}(m+1)^{1 / h} \leq L\left\|A_{m}\right\|\left\|H_{m}^{-1}\right\| g\left(J_{m} A_{m}^{-1} I_{m} H_{m} Q_{m}\right) \\
\Rightarrow & \frac{1}{\eta}(m+1)^{1 / h} \leq L C\left\|A_{m}\right\|\left\|H_{m}^{-1}\right\|\left\|J_{m} A_{m}^{-1} I_{m} H_{m} Q_{m}\right\| \\
\Rightarrow & \frac{1}{\eta}(m+1)^{1 / h} \leq L C\left\|A_{m}\right\|\left\|H_{m}^{-1}\right\|\left\|J_{m} A_{m}^{-1}\right\| \\
& \cdot\left\|I_{m}\right\|\left\|H_{m} Q_{m}\right\|=L C\left\|A_{m}\right\|\left\|H_{m}^{-1}\right\|\left\|A_{m}^{-1}\right\| \\
& \cdot\left\|I_{m}\right\|\left\|H_{m}\right\| \Rightarrow \frac{1}{\eta}(m+1)^{1 / h} \leq 4 L C,
\end{aligned}
$$

for some $L \geq 1$. Since $m$ is an arbitrary, this gives a contradiction. Therefore, $\operatorname{dim}(X)<\infty$ and $\operatorname{dim}(Y)<\infty$ when $S_{V(\gamma, p)}^{\text {app }}$ $(X, Y)=L(X, Y)$. This finishes the proof.

We introduce the next theorem without verification; these can be set up utilizing standard procedure.

Theorem 31. The prequasi Banach operator ideal $S_{V(\gamma, p)}^{K o l}$ is small, whenever $X, Y$ are infinite dimensional Banach spaces and the conditions (a1) and (a2) are satisfied.

Corollary 32. The prequasi Banach operator ideal $S_{c e s}^{a p p}$ is small, if $X, Y$ are infinite dimensional Banach spaces and $p$ $\in(1, \infty)$.

Corollary 33. The prequasi Banach operator ideal $S_{c e s}^{K o l}$ is small, if $X, Y$ are infinite dimensional Banach spaces and $p$ $\in(1, \infty)$.

We examine the sufficient conditions so that the prequasi Banach operator ideal $S_{V(\gamma, p)}$ is simple.

Theorem 34. If $X, Y$ are infinite dimensional Banach spaces, $\left(p_{j}\right) \in \ell_{\infty},\left(q_{j}\right) \in \ell_{\infty}$ with $1<p_{j}<q_{j}$, and $1 \leq \gamma_{j} \leq v_{j}$ for each $j \in \mathbb{N}$, it is true that

$$
\Psi\left(S_{V(v, q)}, S_{V(\gamma, p)}\right)=L\left(S_{V(v, q)}, S_{V(\gamma, p)}\right)
$$

Proof. Let the sufficient conditions be given, $T \in L\left(S_{V(v, q)}\right.$, $\left.S_{V(\gamma, p)}\right)$ and $T \notin \Psi\left(S_{V(v, q)}, S_{V(\gamma, p)}\right)$. From Lemma 1, we have $A \in L\left(S_{V(v, q)}\right)$ and $B \in L\left(S_{V(\gamma, p)}\right)$ with $B T A I_{k}=I_{k}$. For each $k$ $\in \mathbb{N}$, we obtain

$$
\begin{aligned}
\left\|I_{k}\right\|_{S_{V(\gamma, p)}} & =\sum_{n=0}^{\infty}\left(\frac{1}{\gamma_{n}} \sum_{i \in G_{n}} s_{i}\left(I_{k}\right)\right)^{p_{n}} \leq\|B T A\|\left\|I_{k}\right\|_{S_{V(v, q)}} \\
& \leq \sum_{n=0}^{\infty}\left(\frac{1}{v_{n}} \sum_{i \in G_{n}} s_{i}\left(I_{k}\right)\right)^{q_{n}} .
\end{aligned}
$$

In view of Theorem 28, we get a contradiction. Therefore, $T \in \Psi\left(S_{V(v, q)}, S_{V(\gamma, p)}\right)$.

Corollary 35. If $X, Y$ are infinite dimensional Banach spaces, $\left(p_{j}\right) \in \ell_{\infty},\left(q_{j}\right) \in \ell_{\infty}$ with $1<p_{j}<q_{j}$, and $1 \leq \gamma_{j} \leq v_{j}$ for each $j \in \mathbb{N}$, it is true that

$$
L_{C}\left(S_{\operatorname{ces}\left(\left(q_{n}\right)\right)}, S_{\operatorname{ces}\left(\left(p_{n}\right)\right)}\right)=L\left(S_{V(v, q)}, S_{V(\gamma, p)}\right)
$$

Theorem 36. The prequasi Banach operator ideal $S_{V(\gamma, p)}$ is simple, if $X, Y$ are infinite dimensional Banach spaces and the conditions (a1) and (a2) are satisfied.

Proof. Let the sufficient conditions be given and there is $T$ $\in L_{C}\left(S_{V(\gamma, p)}\right)$ with $T \notin \Psi\left(S_{V(\gamma, p)}\right)$. From Lemma 1, we have $A, B \in L\left(S_{V(\gamma, p)}\right)$ so that $B T A I_{k}=I_{k}$. This means that $I_{S_{V(\gamma, p)}}$ $\in L_{C}\left(S_{V(\gamma, p)}\right)$. Consequently, $L\left(S_{V(\gamma, p)}\right)=L_{C}\left(S_{V(\gamma, p)}\right)$. Therefore, $S_{V(\gamma, p)}$ is simple.

\section{Eigenvalues of s-Type Operators}

We investigate the strict inclusion relation between $S_{V(\gamma, p)}$ and $S_{E}^{\lambda}$ (the class of all bounded linear operators whose sequence of eigenvalues belongs to $V(\gamma, p)$ ).

Notations 37. $S_{E}^{\lambda}:=\left\{S_{E}^{\lambda}(X, Y) ; X\right.$ and $Y$ are Banach Spaces $\}$, where $S_{E}^{\lambda}(X, Y):=\left\{T \in L(X, Y):\left(\lambda_{i}(T)\right)_{n=0}^{\infty} \in E\right.$ and $\| T-\lambda_{n}$ $(T) I \|^{-1}$; does not exist for every $\left.n \in \mathbb{N}\right\}$.

Theorem 38. If $X, Y$ are infinite dimensional Banach spaces and conditions (a1) and (a2) are satisfied, it is true that

$$
S_{V(\gamma, p)}(X, Y) \varsubsetneqq S_{V(\gamma, p)}^{\lambda}(X, Y) .
$$

Proof. Let the sufficient conditions be verified and $T \in S_{V(\gamma, p)}$ $(X, Y)$. Therefore, $\left(s_{n}(T)\right)_{n=0}^{\infty} \in V(\gamma, p)$. Hence, we have

$$
\sum_{r=0}^{\infty}\left(\frac{1}{\gamma_{r}} \sum_{k \in G_{r}} s_{k}(T)\right)^{p_{r}} \geq \sum_{r=0}^{\infty}\left[s_{r}(T)\right]^{p_{r}} .
$$

Hence, $\left(s_{r}(T)\right)_{r=0}^{\infty} \in \ell_{\left(p_{r}\right)}$, so $\lim _{r \rightarrow \infty} s_{r}(T)=0$. Assume $\left\|T-s_{r}(T) I\right\|^{-1} \quad$ exists for every $r \in \mathbb{N}$. Therefore, $\left\|T-s_{r}(T) I\right\|^{-1}$ exists and bounded for every $r \in \mathbb{N}$. This gives $\lim _{r \rightarrow \infty}\left\|T-s_{r}(T) I\right\|^{-1}=\|T\|^{-1}$ exists and bounded. From the prequasi operator ideal of $\left(S_{V(\gamma, p)}, g\right)$, we obtain 


$$
\begin{aligned}
I & =T T^{-1} \in S_{V(\gamma, p)}(X, Y) \Rightarrow\left(s_{r}(I)\right)_{r=0}^{\infty} \\
& \in V(\gamma, p) \Rightarrow \lim _{r \rightarrow \infty} s_{r}(I)=0 .
\end{aligned}
$$

We have a contradiction, since $\lim _{r \rightarrow \infty} s_{r}(I)=1$. Therefore, $\left\|T-s_{r}(T) I\right\|=0$ for every $r \in \mathbb{N}$. Hence, $\left(s_{r}(T)\right)_{r=0}^{\infty}$ is the eigenvalues of $T$. Finally, if we choose $\left(s_{r}(T)\right)_{r=0}^{\infty}$ such that $\sum_{k \in G_{r}} s_{k}(T)=\gamma_{r}(r+1)^{-1 / p_{r}}$, we find $T \in L(X, Y)$ such that $\sum_{r=0}^{\infty}\left(1 / \gamma_{r} \sum_{k \in G_{r}} s_{k}(T)\right)^{p_{r}}=\sum_{r=0}^{\infty} 1 /(r+1)=\infty$ and if we take $\left(\lambda_{r}(T)\right)_{r=0}^{\infty}$ such that $\sum_{k \in G_{r}} \lambda_{k}(T)=\gamma_{r} /(r+1)$. Hence, $T \notin$ $S_{V(\gamma, p)}(X, Y)$ and $T \in S_{V(\gamma, p)}^{\lambda}(X, Y)$. This finishes the proof.

\section{Data Availability}

No data were used to support this study.

\section{Conflicts of Interest}

The authors declare that they have no competing interests.

\section{Authors' Contributions}

All authors contributed equally to the writing of this paper. All authors read and approved the final manuscript.

\section{Acknowledgments}

This work was funded by the University of Jeddah, Saudi Arabia (under grant no. UJ-02-058-DR). The authors, therefore, acknowledge with thanks the university technical and financial support.

\section{References}

[1] A. Pietsch, Eigenvalues and S-Numbers, Cambridge University Press, New York, NY, USA, 1986.

[2] R. K. Singh and A. Kumar, "Multiplication operators and composition operators with closed ranges," Bulletin of the Australian Mathematical Society, vol. 16, no. 2, pp. 247-252, 1977.

[3] M. B. Abrahmse, "Multiplication operators," in Lecture Notes in Mathematics, pp. 17-36, 693 Springer Verlag, 1978.

[4] K. Raj, S. K. Sharma, and A. Kumar, "Multiplication operators on Musielak-Orlicz spaces of Bochner type," Journal of Advanced Studies in Topology, vol. 3, no. 4, pp. 1-7, 2012.

[5] A. Sharma, K. Raj, and S. K. Sharma, "Products of multiplication composition and differentiation operators from $H^{\infty}$ to weighted Bloch spaces," Indian Journal of Mathematics, vol. 54, pp. 159-179, 2012.

[6] R. K. Singh and J. S. Manhas, Composition Operators on Function Spaces, North Holland, 1993.

[7] H. Takagi and K. Yokouchi, "Multiplication and composition operators between two $L_{p}$-spaces," Contemporary Mathematics, vol. 232, pp. 321-338, 1999.

[8] M. Mursaleen and A. K. Noman, "Compactness of matrix operators on some new difference sequence spaces," Linear Algebra and its Applications, vol. 436, no. 1, pp. 41-52, 2012.

[9] B. S. Komal and S. Gupta, "Multiplication operators between Orlicz spaces," Integral Equations and Operator Theory, vol. 41, no. 3, pp. 324-330, 2001.
[10] B. S. Komal, S. Pandoh, and K. Raj, "Multiplication operators on Cesàro sequence spaces," Demonstratio Mathematica, vol. 49, no. 4, 2016.

[11] A. Pietsch, Operator Ideals, North-Holland Publishing Company, Amsterdam-New York-Oxford, 1980, MR582655 (81j:47001).

[12] A. Pietsch, "Small ideals of operators," Studia Mathematica, vol. 51, no. 3, pp. 265-267, 1974.

[13] B. M. Makarov and N. Faried, Some Properties of Operator Ideals Constructed by S Numbers (in Russian), Theory of Operators in Functional Spaces, Academy of Science, Siberian section, Novosibirsk, Russia, 1977.

[14] A. A. Bakery, "Mappings of type generalized de La Vallée Poussin's mean," Journal of Inequalities and Applications, vol. 2013, no. 1, 2013.

[15] S. A. Mohiuddine and K. Raj, "Vector valued Orlicz-Lorentz sequence spaces and their operator ideals," The Journal of Nonlinear Sciences and Applications, vol. 10, no. 2, pp. 338353, 2017.

[16] N. Faried and A. A. Bakery, "Small operator ideals formed by $s$ numbers on generalized Cesáro and Orlicz sequence spaces," Journal of Inequalities and Applications, vol. 2018, no. 1, 2018.

[17] T. Mrowka, A Brief Introduction to Linear Analysis: Fredholm Operators, Fall, Geometry of Manifolds, 2004, (Massachusetts Institute of Technology: MIT OpenCouseWare).

[18] N. Simsek, E. Savas, and V. Karakaya, "Some geometric and topological properties of a new sequence space defined by de la Vallée-Poussin mean," Journal of Computational Analysis and Applications, vol. 12, no. 4, pp. 768-779, 2010.

[19] L. Leindler, "Über die verallgemeinerte de la ValléePoussinsche summierbarkeit allgemeiner Orthogonalreihen," Acta Mathematica Academiae Scientiarum Hungaricae, vol. 16, no. 3-4, pp. 375-387, 1965.

[20] I. J. Maddox, “On Kuttners theorem," Journal of the London Mathematical Society, vol. 43, pp. 285-290, 1968.

[21] W. Sanhan and S. Suantai, "On k-nearly uniform convex property in generalized Cesàro sequence spaces," International Journal of Mathematics and Mathematical Sciences, vol. 2003, no. 57, 3607 pages, 2003.

[22] P. Y. Lee, "Cesáro sequence spaces," Mathematical Chronicle, vol. 13, pp. 29-45, 1984.

[23] Y. Q. Lui, B. E. Wu, and Y. P. Lee, Method of Sequence Spaces, Guangdong of Science and Technology Press, 1996.

[24] J. S. Shiue, "On the Cesàro sequence spaces," Tamkang Journal of Mathematics, vol. 1, no. 1, pp. 19-25, 1970.

[25] A. A. Bakery and M. M. Mohammed, "Small pre-quasi Banach operator ideals of type Orlicz-Cesáro mean sequence spaces," Journal of Function Spaces, vol. 2019, Article ID 7265010, 9 pages, 2019.

[26] B. Altay and F. Başar, "Generalization of the sequence space $\ell(\mathrm{p})$ derived by weighted mean," Journal of Mathematical Analysis and Applications, vol. 330, no. 1, pp. 174-185, 2007.

[27] A. Pietsch, Operator Ideals, VEB Deutscher Verlag derWissenschaften, Berlin, 1978.

[28] A. Pietsch, "s-numbers of operators in Banach spaces," Studia Math, vol. 51, no. 3, pp. 201-223, 1974. 